\title{
Editorial
}

\section{All's well that ends well: translational control and cell death}

Cell Death and Differentiation (2005) 12, 533. doi:10.1038/sj.cdd.4401656

This special issue is devoted to the mechanisms used by apoptosing cells in order to maintain the expression of essential apoptotic proteins. There has been a great deal of interest in this area since the late 1990s, when the laboratories of Mike Clemens, ${ }^{1}$ Simon Morley ${ }^{2}$ and Rick Lloyd ${ }^{3}$ demonstrated that during apoptosis there is a considerable reduction in the rate of protein synthesis. This decrease is in part the consequence of the targeted proteolytic cleavage by caspase3 of eukaryotic initiation factor 4G (elF4G), the large bridging protein that contains binding sites for elF4A (a DEAD-box helicase), elF4E (the mRNA cap binding protein) and elF3 (which is involved in ribosome recruitment). Subsequent studies have shown that other modifications to the translational machinery and associated proteins also occur during apoptosis, notably the cleavage of elF4B, elF $2 \alpha, 4 \mathrm{EBP} 1$ and the P35 subunit of elF $3,{ }^{4}$ and also changes in the phosphorylation states of elF2 $\alpha$ and 4EBP1.,6 Some of these aspects are highlighted in manuscripts by Mike Clemens, Simon Morley and Chris Proud in this issue.

When apoptosis is induced (by a variety of agents), protein synthesis is reduced to between 10 and $30 \%$ of the level in control cells. This suggests that the subset of messages that continue to be expressed are using an alternative mechanism of translation. Establishing quite what this mechanism is that enables proteins synthesis in apoptotic cells is therefore of prime importance to those in the translation field.

It is now well established that there are two mechanisms that are used for translation initiation in eukaryotic cells: capdependent scanning, which is dependent upon the canonical initiation factors assembling at the $5^{\prime}$ cap, and internal ribosome entry where the ribosome is recruited to a complex structural RNA element known as an internal ribosome entry segment (IRES).

IRES-mediated translation is able to occur under conditions where cap-dependent scanning is inhibited, and can act as a molecular switch allowing translation of certain subsets of mRNAs under stress conditions including apoptosis. In agreement with this, a number of studies have shown that certain IRESs are active during apoptosis, and are consequently able to maintain the expression of proteins that play a part in the apoptotic process, including c-myc, ${ }^{7} \mathrm{XIAP}^{8}$ and DAP5. ${ }^{9}$ The roles of certain IRESs in regulating expression of proteins during apoptosis is discussed in this issue by Martin Holcik and Adi Kimchi.

It is becoming apparent that the regulation of IRESs during apoptosis is likely to be brought about by the IRES transacting factors (ITAFs) that interact with the $5^{\prime}$-UTRs of apoptotic messages. This immediately suggests a mechanism by which such proteins could allow the coordinated regulation of subsets of IRESs under certain conditions. Very few ITAFs have been identified to date, and the role of these proteins in regulating IRES function is discussed in this issue in a manuscript by Spriggs et al.

Finally, we know that aberrant translational regulation of certain proteins, particularly those required for apoptosis, is associated with disease (especially neoplasias). This topic will be addressed in this issue in articles by Glen Barber and Danilo Perroti.

\footnotetext{
AE Willis *,1

1 School of Pharmacy, University of Nottingham, University Park, Nottingham NG72RD, UK

* Corresponding author: AE Willis, School of Pharmacy, University of Nottingham, University Park, Nottingham NG72RD, UK

E-mail: anne.willis@nottingham.ac.uk

1. Clemens MJ et al. (1998) Oncogene 17: 2921-2931

2. Morley SJ et al. (1998) FEBS Lett. 438: 41-48

3. Marissen WE and Lloyd RE (1998) Mol. Cell. Biol. 18: 7565-7574

4. Clemens MJ et al. (2000) Cell Death Differ. 7: 603-615

5. Bushell $\mathrm{M}$ et al. (2000) Eur. J. Biochem. 267: 1083-1091

6. Tee AR and Proud CG (2002) Mol. Cell. Biol. 22: 1674-1683

7. Stoneley M et al. (2000) Mol. Cell. Biol. 20: 1162-1169

8. Holcik M et al. (1999) Nat. Cell Biol. 1: 190-192

9. Henis-Korenblit S et al. (2000) Mol. Cell. Biol. 20: 496-506
} 